\title{
La Volksbühne des années 1970 : une institution rebelle à Berlin-Est
}

Maurice Taszman, entretien réalisé par Charlotte Bomy. Berlin, 9 septembre 2012

Die Volksbühne der 1970er Jahre: eine Ostberliner Institution probt den Aufstand The Volksbühne in the 1970s: A Rebel Institution in East Berlin

Charlotte Bomy et Maurice Taszman

\section{CpenEdition Journals}

Édition électronique

URL : https://journals.openedition.org/ceg/9022

DOI : $10.4000 /$ ceg. 9022

ISSN : 2605-8359

Éditeur

Presses Universitaires de Provence

Édition imprimée

Date de publication : 1 mai 2013

Pagination : 323-334

ISBN : 0751-4239

ISSN : 0751-4239

Référence électronique

Charlotte Bomy et Maurice Taszman, « La Volksbühne des années 1970 : une institution rebelle à Berlin-Est », Cahiers d'Études Germaniques [En ligne], 64 | 2013, mis en ligne le 02 janvier 2020, consulté le 02 juin 2021. URL : http://journals.openedition.org/ceg/9022 ; DOI : https://doi.org/10.4000/ceg. 9022 


\title{
La Volksbühne des années 1970 : une institution rebelle à Berlin-Est
}

\author{
Maurice Taszman, entretien réalisé par Charlotte Bomy \\ Berlin, 9 septembre 2012
}

Maurice Taszman est né en 1934 à Bruxelles. Durant les cinquante dernières années, il a travaillé comme réalisateur, auteur, traducteur, dramaturge et conseiller littéraire dans plusieurs théâtres, principalement en France, en Allemagne et en Belgique. Dès 1969, il enseigne à Berlin-Ouest la pratique du cinéma documentaire à la Deutsche Film- und Fernsehakademie Berlin - une institution typiquement berlinoise, régulièrement agitée par les turbulences politiques de la fin des années $1960^{1}$.

En 1972, à l'occasion de la préparation de la mise en scène collective de la pièce Le Neveu de Rameau d'après Diderot ${ }^{2}$, il rejoint l'équipe de la Volksbühne-Ost où il devient par la suite dramaturge et conseiller artistique. Il reste à Berlin-Est jusqu'au départ de Benno Besson en 1977. C'est en tant que grand témoin d'une époque d'effervescence artistique dans la capitale allemande, mais aussi en tant que passeur ou médiateur culturel et contreculturel, entre Berlin-Est et Berlin-Ouest, qu'il est intervenu à Toulouse durant la journée d'études de novembre 2011 et le colloque de février 2012 consacrés aux contre-cultures théâtrales.

Charlotte Bomy : En réfléchissant à la notion de contre-culture, nous nous sommes interrogés sur la possibilité de trouver quelque chose de contreculturel du côté de Berlin-Est, dans un pays où la culture, et le théâtre en particulier, était adossés à une doctrine visant la stabilisation de l'État socialiste et ne supposant pas le développement d'un modèle culturel pluriel

1 Voir à ce sujet les articles de Jeremy Hamers et André Combes dans le présent numéro.

2 Le spectacle Rameaus Neffe (Le Neveu de Rameau) fut présenté pour la première fois le 3 décembre 1973 à la Volksbühne-Ost, dans une mise en scène collective réalisée par Brigitte Soubcyran, Ernstgcorg Hcring, Mauricc Taszman, Hclmut Straßburger, Klaus Waack. La spécificité de l'entreprise fut que les mettcurs en scène avaient reconstituć le Café de la Régence dans un des foyers du théâtre, les spectatcurs étaient attablés ct les comédiens circulaient à travers les tables. Le spectacle proposait une forme de convivialité en soirée qui était rare à Berlin-Est, ce qui lui valut d'être à l'affíche pendant plus de 10 ans. 
et critique comme en Allemagne de l'Ouest. La Volksbühne des années 1970, celle de l' " ère Benno Besson » semble pourtant avoir connu une existence relativement autonome et critique. Que représente pour toi la Volksbühne de cette époque?

Maurice Taszman : D'une certaine façon, la Volksbühne a toujours été une figure de proue et un vestige actif de l'histoire contre-culturelle allemande. Lorsqu'elle fut "fondée » en $1914^{3}$, selon une logique coopérative, les ouvriers cotisaient pour avoir un accès à une culture qui leur était jusque-là interdite ${ }^{4}$. La social-démocratie allemande dans ce qu'elle avait de meilleur souhaitait que les couches populaires ne soient plus éloignées du théâtre. Plusieurs Volksbühnen furent d'ailleurs créées en Allemagne à cette époque afin que chaque ville puisse s'honorer d'avoir un thêâtre du peuple, mais aucune d'elle n'a véritablement remis en question l'esthétique du théâtre bourgeois. À la Volksbühne de Berlin, en revanche, des personnalités comme Piscator qui y était metteur en scène associé entre 1924 et 1927 ont révolutionné le théâtre : avec un théâtre créateur de formes et d'architectures, en même temps qu'il faisait fonction d'outil politique. Ce fut l'une des grandes périodes de la Volksbühne, avant que celle-ci ne passe entre les mains de l'administration nazie. Après 1945, elle était un théâtre de grande consommation, avec un répertoire classique, basé sur le star system, si ce n'est pendant la direction de Fritz Wisten ${ }^{5}$. Avec l'arrivée de Besson comme directeur en $1969^{6}$, la Volksbühne de Berlin-Est s'est transformée à nouveau en un lieu propice aux expérimentations artistiques. Besson est arrivé là après avoir été « chassé » du Berliner Ensemble où il avait été auparavant acteur et metteur en scène. Mais ses mises en scène les plus importantes avaient été réalisées au Deutsches Theater. Brecht lui faisait une confiance énorme : il lui avait d'ailleurs confié l'ouverture du Theater am Schiffbauerdamm ${ }^{7}$ avec une mise en scène de Dom Juan. Besson était l'élève «bohème » de Brecht; à ce titre, il voulait renouer avec un théâtre populaire. Il a revu l'architecture intérieure de la Volksbühne, en faisant démonter les loges et en mettant les places au même niveau dans une salle en pente. Il a également supprimé les abonnements qui « débarrassèrent » le théâtre des officiels du régime - ce qui

3 Fondée en 1890, la Volksbühne connaît en 1892 une seission entre la Freic Volksbühne et la Ncue Frcic Volksbühnc. C'cst la construction du théâtre sur l'cmplacement actucl qui date de 1913-1914.

4 Selon le mot d'ordre de la culture SPD : «Die Kunst dem Volke ».

5 Rescapé des camps, le comédien juif autrichien Fritz Wisten (1890-1962) a dirigé la Volksbühnc de 1953 à 1961. Il avait auparavant été à la tête du Deutsches Theater ct du Theater am Schiffbauerdamm.

6 Benno Besson a d'abord ćté nommé directcur artistique (1969-1974), puis directeur du théâtre (Intendant) de 1974 à 1977.

7 Rouvert après la Scconde gucrre mondialc, le Deutsches Nationaltheater am Schiffbaucrdamm dirigé par Wolfgang Langhoff de 1946 à 1963, accucillc lc Berlincr Ensemble de Brecht et Helene Weigel entre 1949, annéc de sa création, ct 1954, annćc où eclui-ci s'installa dans le Theater am Schiffbauerdamm. 
a sans doute favorisé le développement d'un espace de liberté dans le théâtre durant ces années-là. Et surtout, pour toucher un large public, Benno Besson a proposé un nouveau type de manifestations, les Spektakel, ces grandes soirées-spectacles où le public était convié à passer une partie de la nuit au théâtre, où les artistes se produisaient simultanément dans tous les espaces de jeu possibles : devant et derrière le rideau de fer (du théâtre, bien sûr !), mais aussi dans la cantine et dans les couloirs du théâtre... Les spectateurs devaient venir quatre jours pour pouvoir assister à la totalité des pièces présentées. Besson a su introduire de la couleur, de la démesure, tout en s'entourant d'une constellation de personnalités fort différentes.

CB : Au même moment, à la Schaubühne de Berlin-Ouest, animée par Peter Stein et Claus Peymann, la notion de collectif était mise en avant pour fédérer une troupe permanente, avec la revendication d'une gestion collective et le souci de construire un nouveau rapport au public. À quoi ressemblait le fonctionnement interne de la Volksbühne?

MT : La notion de co-gestion (Mitbestimmung) n'existait pas comme dans le fondement de la Schaubühne ${ }^{8}$. Ce n'était pas un modèle discuté, car le fonctionnement était très différent. On pouvait parler d'un fonctionnement collégial, mais qui ne se voulait pas démocratique : le théâtre devait rester un lieu de conflit, où chacun devait garder son identité et son activité critique. Par rapport aux autres théâtres de Berlin-Est, jamais la Volksbühne ne s'est appelée « Ensemble » - c'était plutôt le modèle du Berliner Ensemble où il y avait eu Brecht, un maître et des disciples. La Volksbühne des années 1970 n'était pas un théâtre avec un maître qui déterminait le répertoire. Elle représentait plutôt une sorte d'héritage critique de Bertolt Brecht, où il s'agissait justement de prendre ces distances d'avec cet héritage, c'était le théâtre des fils révoltés. Besson voulait autour de lui des gens qui ne fassent pas la même chose, tout en étant des personnalités fortes avec des compétences et des projets esthétiques différents. D'une certaine manière, en devenant chef de troupe, il a renoncé à ses propres ambitions artistiques développées au Deutsches Theater pour monter des pièces à succès (Molière, Offenbach), ce qui permettait à d'autres d'expérimenter. Il a proposé à des metteurs en scène comme Matthias Langhoff et Manfred Karge, à l'époque personae non gratae au Berliner Ensemble, de s'associer au théâtre. Il y avait également Brigitte Soubeyran, formée à la pantomime et qui avait déjà travaillé avec Besson au Deutsches Theater dans les années 1960 : elle a monté un spectacle avec Einar Schleef comme scénographe ${ }^{9}$ qui fit ainsi ses débuts à la Volksbühne-Ost. Fritz Marquardt faisait aussi partie de l'équipe, avec une recherche esthétique plus tournée vers le théâtre soviétique des

8 À ce sujet, consulter la contribution de Catherine Mazcllier-Lajarrige dans ce volume.

9 Il s'agit du spectaclc Don Gil von den grünen Hosen (Don Gil de vert vêtu) d'après Tirso de la Molina, mise en seène de Brigitte Soubcyran et présenté pour la première fois en 1972 à la Volksbühne-Ost. 
années 1920-1930. On le connaît peu en France, mais il avait mis en scène deux pièces de Heiner Müller : Der Bau (La Construction) et Die Bauern (Les Paysans) - pour cette dernière, il s'agissait en fait de Die Umsiedlerin ( $\mathrm{La}$ Déplacée), interdite en RDA peu de temps après sa création en 1961, dont Müller avait simplement changé le titre! Heiner Müller était bien sûr l'une des grandes personnalités du théâtre, c'était l'auteur associé : un auteur maison qui n'était pas en odeur de sainteté depuis son exclusion de l'Union des écrivains en 1961.

CB : Venons-en à un projet théâtral singulier et marginal de la Volksbühne en 1976, documenté par un film à la réalisation duquel tu as participé : le séminaire dirigé par Besson autour de L'Exception et la règle ${ }^{10}$ de Brecht, avec des ouvriers d'usines de Berlin-Est. Comment est née l'idée d'une telle formation?

MT : Ce projet a débuté en Italie. À cette époque, Besson allait souvent en Italie pour monter du Brecht, puisque ce n'était pas possible à Berlin. Brecht demeurait l'apanage et le monopole du Berliner Ensemble, il y avait toujours de nombreuses discordes et batailles avec les héritiers de Brecht. Au cours de ses répétitions au Teatro Argentina, Besson avait reçu un jour un appel d'un éminent membre du parti communiste italien, Giorgio Napolitano, qui lui avait exposé son problème : le vote d'une nouvelle convention collective permettait désormais d'allouer 150 heures à destination des travailleurs dans les domaines de la chimie et de la métallurgie, pour que ceux-ci aient enfin accès à la culture. Mais que proposer durant ces heures de formation continue, payées par le patronat ? Les Italiens se sont tournés vers Luigi Nono, Dario Fo, Benno Besson et quelques autres pour faire des propositions. C'est comme ça que Besson est parti - avec Karge et Langhoff - à Terni, en Ombrie, mener un atelier sur le Lehrstück pour des ouvriers. Besson voulait interroger la validité et l'actualité du Lehrstück. Un an après, il a poursuivi cette expérience de séminaire autour du Lehrstück, mais cette fois avec les ouvriers d'une usine de Berlin-Est. L'atelier de jeu avait lieu dans l'usine même, c'était une idée qui plaisait à Besson. Tout au début du film, on voit bien qu'on se trouve à l'intérieur d'une usine : à travers le vitrage, de l'autre côté de la cour, il y a des gens qui travaillent à la fabrication de lampes. Besson le souligne, il dit qu'il tient à ce qu'on s'aperçoive que la culture n'est possible que si d'autres gens produisent quelque chose dans le même temps : cette dimension, la division travail/théâtre, est inscrite dans l'architecture du film.

10 Die Ausnahme und die Regel (L'Exception et la règle) de Brecht, séminairc sous la direction de Benno Besson, 9-27 février 1976. Le film documentaire sur ce projet est disponible aux archives de l'Akademic der Künste à Berlin. 
CB : En somme, le même programme s'est d'abord déroulé dans un pays capitaliste avec des ouvriers métallurgistes communistes (en Italie), puis dans un pays du «socialisme réellement existant» (la RDA). Le séminaire de Besson, Karge et Langhoff est en ce sens un projet contre-culturel, porté par l'institution (la Volksbühne) mais produit extra muros, dans l'usine. C'est en tout cas une expérience unique dans les annales du théâtre politique estallemand d'après 1945. Quel impact didactique ce Lehrstück a-t-il pu avoir pour les ouvriers de RDA ?

MT : L'implication des participants était essentielle dans cette formation culturelle. Le Lehrstück - que je préfère traduire en français par «pièce d'apprentissage » que par "pièce didactique »-a été développé par Brecht à la fin des années 1920 et au début des années 1930. Après le succès de l'Opéra de quat'sous, il s'était rendu compte qu'en travaillant pour l'institution, il restait prisonnier d'un théâtre «culinaire ». Il lui fallait donc se tourner vers un autre dispositif. Le Lehrstück, comme cela est dit au début du film par Besson, n'est pas fait pour être vu (comme le Schaustück), c'est quelque chose qui est fait pour être joué. En même temps, c'est une utopie concrète, car on ne s'improvise pas comédien ou metteur en scène, ce sont des métiers. Ce qu'on peut faire avec les ouvriers, c'est travailler à des transferts de savoirs, tout en vérifiant leur validité. Grâce au Lehrstück, on peut produire une nouvelle forme de savoir culturel. En jouant un certain nombre de rôles différents, les participants circulent dans ces rôles et apprennent des choses sur ce que dit le texte de la pièce, et par extension sur la réalité qu'ils vivent, sur la manière dont cette réalité est saisissable. On voit dans le film que, dans la salle où se déroule la formation, les gens sont assis en cercle, il n'y a pas de rapport magistral, tout le monde est mélangé et à la même hauteur. La salle et la scène sont intégrées dans un dispositif opératoire : les acteurs deviennent spectateurs, et vice-versa. L'intervention de la réalité passe par la participation active des spectateurs. Les pièces d'apprentissage sont de véritables propositions de jeu et de réflexion, ce sont, pour reprendre les mots de Brecht dans un entretien, des «exercices d'assouplissement destinés à ces sortes d'athlètes de l'esprit que doivent être les bons dialecticiens $»^{11}$.

11 Bertolt BRECHT à Pierre ABRAHAM sur La Décision dans Europe, «Brecht», $\mathrm{n}^{\circ}$ 133-134, janvier-février 1957, p. 173. 


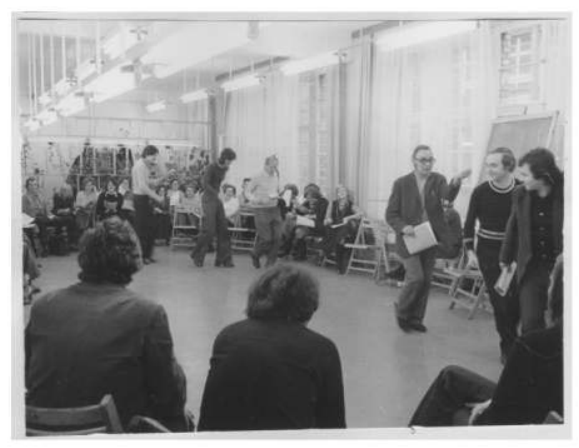

Sous le regard de Besson et de leurs collègues de travail, les acteurs ouvriers de L'Exception et la règle transforment une salle de réunion en salle de travail théâtral.

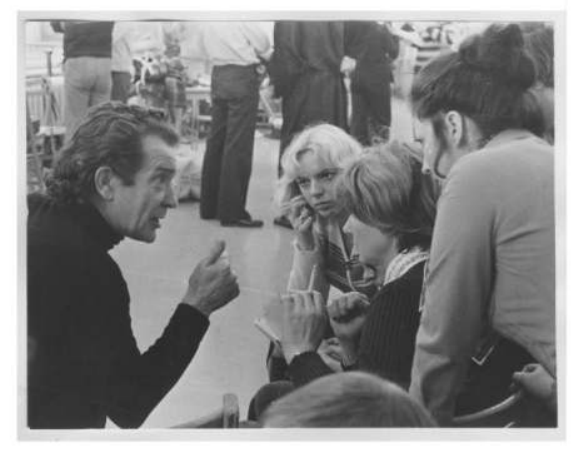

Benno Besson donne des explications à un groupe d'ouvrières dans la salle.

Parole et regard - la pièce d'apprentissage brechtienne revivifiée par des ouvrières et ouvriers de Berlin-Est. Atelier de Benno Besson sur L'Exception et la règle de Brecht (1976). Akademie der Künste, Sammlung Inszenierungsdokumentationen Nr. 162. (C) Antje Stötter.

CB : On peut compléter au sujet du Lehrstück en RDA avec ce que mettait en avant André Combes - qui fut aussi témoin de cette époque - durant la journée d'études de novembre 2011. Il évoquait l'importance de l'ouvrage de Reiner Steinweg sur le Lehrstück ${ }^{12}$ en Allemagne de 1'Ouest, notamment diffusé par la revue littéraire marxiste berlinoise alternative, qui était très lue dans les milieux intellectuels des années 1970. Steinweg insiste sur le fait que le Lehrstück appartient à une société future, socialiste, avec un nouveau mode de production du théâtre qui n'aura plus rien à voir avec la division scènesalle et la division acteur-public, et qui fera en sorte que le collectif soit un espace de circulation des acteurs, des personnages et des rôles, ce qui pouvait signifier revisiter des structures très anciennes (protagonistes/antagonistes/ chœurs). Cette relecture de Brecht en RFA a eu un impact sur la théorie théâtrale en RDA et l'évolution du brechtisme. Des théoriciens comme Werner Mittenzwei vont se « repencher » sur le Lehrstück aux alentours de 1972 et 1975, sous l'impulsion de Steinweg - qui, lui, était issu du mouvement étudiant de Berlin-Ouest et voyait le Lehrstück comme un théâtre d'intervention dans des milieux spécifiques (écoliers, apprentis, ouvriers par ex.) - mettant l'accent sur la production plutôt que sur la réception, et praticable par tout le monde. Mittenzwei, qui lui reprochait au début, dans l'orbe de Lukács, de ne pas saisir la société dans la totalité par une dialectique

12 Reiner STEINWEG, Das Lehrstück. Brechts Theorie einer politisch-ästhetischen Erziehung, Stuttgart, Metzler, 1972. 
de l'individuel et du collectif, comprend que l'objectif pédagogique principal du Lehrstück est l'apprentissage de la dialectique in concreto. C'est donc également une autre forme de transfert contre-culturel, cette fois-ci théorique, passant de l'Allemagne de l'Ouest à l'Allemagne de l'Est qui peut expliquer l'intérêt du projet de Besson dans cette usine.

Plus concrètement, que faisiez-vous avec le texte de L'Exception et la règle ? Comment se déroulaient vos journées de travail pendant la formation?

MT : Le séminaire débutait dans l'usine à 8 heures du matin, on commençait par chanter. Le chant rassemble, il permet de rentrer dans une forme de collectivité. C'est Paul Dessau qui a composé la musique de L'Exception et la règle, mais c'est un aspect que Besson ne reprenait pas dans l'atelier. En revanche, il voulait réintégrer les chœurs, les faire jouer et chanter par le public du séminaire. En faisant des recherches dans les archives de Brecht, où il y a plusieurs variantes de L'Exception et la règle, Karge et Langhoff avaient découvert une version où le récit était commenté par un « chœur de droite» et « un chœur de gauche ». Le chœur de droite affirme qu'il faut faire vite pour le progrès de l'humanité, alors que le chœur de gauche dit au coolie: "Freine, freine, tu vas y laisser ta peau!" Avec Besson, ils avaient décidé de réintégrer cette idée, qui interpellait directement l'idéologie dominante productiviste en RDA, dans le séminaire. On jouait également tout une série de petites scènes, sans cesse interrompues par Besson qui demandait aux participants de faire une description de ce qu'ils avaient vu. Il fallait exclure tout jugement de valeur sur la qualité du jeu, et penser à ce qui se passait, ce qui se faisait. Les gens sont ce qu'ils font, non pas ce qu'ils disent. Il y avait sans cesse des passages du jeu à la réflexion. Dans L'Exception et la règle, comme dans d'autres pièces de Brecht, il est question de bonté, de la difficulté qu'il y a à être bon dans un monde mauvais, capitaliste. C'est une pièce qui avance vite, dans l'ironie et la cruauté, avec des scènes brèves construites en rupture les unes par rapport aux autres : c'est une « machine à jouer». La pièce raconte l'histoire d'un marchand, qui doit traverser un désert pour conclure une affaire, accompagné d'un guide et d'un pauvre coolie. Le marchand se débarrasse du guide car il trouve que celui-ci prend trop d'ascendant sur le coolie. Il y a trop de jonctions entre ces deux subordonnés, donc un risque d'inconnu, c'est une situation de crise pour le marchand schizophrène. Il finit par se sentir menacé par le coolie également et le tue. Comme il n'y a aucun témoin, il est déclaré non coupable. Dans le cadre du séminaire à Berlin-Est, la pièce de Brecht permettait d'explorer dialectiquement l'univers du travail, les rapports de la culture du travail - et pas seulement dans un monde capitaliste, mais aussi dans une société où les rapports marchands sont prétendument abolis, où l'on peut aussi subir une forme d'exploitation ouvrière et d'exclusion, où des antagonismes demeurent. 
CB : Est-ce que cette expérience est d'une certaine manière représentative du paradoxe de l'héritage brechtien en RDA?

MT : L'héritage de Brecht était, je crois, très problématique des deux côtés du Mur. Dans les années 1950, une question se posait : quel état allemand était le mieux à même d'assurer l'héritage brechtien ? Hanns Eisler et Ernst Bloch ont écrit un texte lors du débat sur 1'expressionnisme qui s'intitule « l'art d'hériter» (1938), car il y a nécessairement des choix dans l'héritage. Chaque état réclamait un héritage différent. En RFA, l'héritage du mouvement prolétarien n'existait pas, il y avait un travail de déblayage à faire à partir des textes essentiels qui avaient été jusque-là exclus de ce qu'on enseignait - c'est ce que prendra en main le mouvement étudiant à partir de 1968, dans une nouvelle conjoncture culturelle. En RDA, déjà du temps de Brecht qui avait choisi d'y travailler, il y avait d'autres aspects qui étaient problématiques. Par exemple, à la création de Mère Courage en 1949 au Deutsches Theater (à l'époque où Brecht n'avait pas encore le Theater am Schiffbauerdamm et où Wolfgang Langhoff lui avait prêté un théâtre), une partie du public était scandalisée par les accents des comédiens : Helene Weigel avait un accent autrichien, Ernst Busch un accent hollandais. Brecht voulait qu'on entende à la scène l'histoire de tous ces gens qui portent cette langue allemande, c'était un théâtre de l'exil qui se rassemblait à nouveau. Il y avait "contre-culture », dans la mesure où cela ne correspondait pas aux vœux des dirigeants est-allemands d'un grand théâtre allemand qui serait celui d'une nation socialiste unifiée. Brecht, lui, souhaitait échapper à l'allemand prussien, il ne voulait pas uniformiser la langue, mais réintroduire des parlers régionaux. Ce qui pose des problèmes de traduction vers le français.

D'ailleurs, l'héritage brechtien était aussi problématique en France: Brecht était considéré comme un auteur « rive gauche », notamment lorsque Jean-Marie Serreau a monté, pour la première fois en France, L'Exception et la règle en 1949. C'est Vilar qui a popularisé véritablement Brecht en France, car ses textes lui permettaient de mettre en œuvre son idée d'un « théâtre citoyen ». Avec les tournées du Berliner Ensemble à Paris, l'arrivée de Brecht en France a été ensuite un moment important pour la théorie théâtrale et la critique, notamment dans les textes de Barthes pour la revue Théâtre populaire.

De manière générale, pour revenir sur l'aspect philologique, le problème Brecht est un vrai casse-tête. C'est aussi parce que pour la totalité de ses pièces, il y a énormément de variantes. Pour Baal, par exemple, il y a 5 versions. L'épreuve du plateau devient alors toujours déterminante, c'était une donnée importante pour Brecht lui-même qui mettait ses propres textes à l'épreuve de la scène.

CB : Pourrais-tu nous donner un autre exemple de ce qui se pratiquait comme contre-culture théâtrale à la Volksbühne ? 
MT : Ce qui s'est également pratiqué à la Volksbühne, moins par Besson que par Karge, Langhoff et Müller, c'était une manière de faire du théâtre en étant extrêmement sensible à la " misère allemande », cet immobilisme et cette servilité, pointés par Engels. Il fallait affronter la misère d'un pays qui n'a pas connu de révolution depuis la «Guerre des Paysans » au XVI ${ }^{\mathrm{e}}$ siècle, qui avait de surcroît été un échec. C'était un terme imprononçable en RDA, cette « misère » était censée avoir disparu. Hanns Eisler avait déjà tenté de se confronter à la misère allemande et à la guerre des Paysans en écrivant son opéra Johann Faustus en 1952, sous 1'influence du Doktor Faustus de Thomas Mann. Mais il n'a jamais pu monter ce texte, car la RDA voulait voir dans Faust le fleuron de l'héritage classique pour installer une nouvelle culture nationale. L'une des plus belles réalisations de Karge et Langhoff, selon moi, a été Der Bürgergeneral (Le Général citoyen) de Goethe où celuici prend position contre la Révolution française. On voyait dans ce spectacle de quelle manière ils s'opposaient à l'idéalisation de Goethe qui était de mise en RDA, ils montraient que Goethe avait également été un philistin et un petit-bourgeois. La " misère allemande » était également évoquée dans leur spectacle Die Räuber (Les Brigands) de Schiller où il ne s'agissait pas de mettre en lumière le conflit entre les frères Karl et Franz (et leur père), mais plutôt de comprendre les motivations, le parcours et l'échec de la bande de brigands.

Un autre spectacle marquant de Karge et Langhoff a été Die Schlacht ( $\mathrm{La}$ Bataille) de Heiner Müller en 1975. Ce texte expose en cinq segments des situations de débâcles familiales dans l'Allemagne en guerre. C'est devenu un spectacle très important car les gens de RDA, avec l'appui des idéologues, en étaient arrivés à croire que le nazisme ne les concernait pas. Ils pensaient qu'ils étaient du bon côté, alliés de l'URSS, et que le nazisme venait de l'Ouest. La culture officielle présentait les Allemands de l'Est comme étant du bon côté de l'Histoire. Avec cette mise en scène, une jeune génération découvrait la réalité de la guerre. Entre chaque fragment et chaque changement de scénographie, des gens qui avaient assisté aux répétitions venaient raconter ce qu'avait été pour eux 1945, ces images de Berlin où à chaque réverbère pendait un cadavre, quelqu'un qui s'était pendu ou bien qui avait été accroché-là par la SS. Le rôle de Müller était très important dans cet impact contre-culturel de la Volksbühne, ses textes permettaient une revisitation de l'espace mémoriel de RDA. Il ne se voulait pas historien, mais il se confrontait à l'Histoire pour débusquer ce que les histoires officielles ne disent pas.

CB : Ceci dit, il n'y avait pas qu'à la Volksbühne qu'on montait du Müller. Pratiquement à la même époque, Ruth Berghaus qui dirigeait le Berliner Ensemble avait aussi réalisé une mise en scène d'un texte de Heiner Müller. 
MT : Oui, mais ça n'avait pas était une mince affaire. C'est vrai que Ruth Berghaus est également une figure très importante de cette époque, une des artistes femmes qui marquèrent la vie artistique et culturelle de Berlin-Est. Elle avait été l'élève de Gret Palucca et était considérée en RDA comme une chorégraphe formaliste. En 1971, à la mort de Helene Weigel, elle est devenue la directrice du Berliner Ensemble et a essayé de régénérer ce théâtre qui, depuis la mort de Brecht en 1956 et surtout depuis la construction du Mur, avait tendance à devenir un musée. Au début des années 1970 en RDA, avec l'arrivée au pouvoir d'Erich Honecker, il y a eu un relatif assouplissement de la politique culturelle, après des années difficiles pour le théâtre. C'est dans ce contexte d'une politique culturelle plus flexible que Ruth Berghaus est parvenue à imposer aux autorités de la RDA sa création de Ciment de Heiner Müller en 1972. Mais cette parenthèse n'a pas duré très longtemps. Entre les polémiques déclenchées par les représentations de Mademoiselle Julie, mise en scène par Bernhard Klaus Tragelehn et Einar Schleef en 1976, et les conflits avec ceux qui se réclamaient de l'héritage de Brecht, il y avait de plus en plus de pression autour de Berghaus qui a été obligée de démissionner en 1977, au profit de Manfred Wekwerth ${ }^{13}$. Avec lui, la famille Brecht reprenait ses droits sur "l'héritage menacé ».

Le problème des héritiers de Brecht nuisait également au développement de la Volksbühne qui souffrait d'être le théâtre de Müller mais de ne pas pouvoir jouer du Brecht. Besson avait uniquement le droit de monter $L a$ Bonne Âme du Se-Tchouan car on avait retrouvé un télégramme de la main de Brecht l'autorisant à le faire! Globalement, le contexte politique culturel était aussi devenu beaucoup plus tendu après l'affaire Biermann en 1976. Des mesures répressives étaient prises contre les intellectuels et les artistes, beaucoup quittèrent la RDA. Manfred Karge et Matthias Langhoff sont partis travailler en Allemagne de l'Ouest en 1976, Besson quant à lui a démissionné en 1977 et est finalement parti à Paris en 1978. Dès lors, je n'avais plus de raison de vivre à Berlin-Est ni d'y travailler.

CB : Il devenait très difficile d'exprimer sa voix en rupture avec l'esthétique dominante en RDA. Les espaces de liberté étaient ailleurs que dans le théâtre officiel. Les auteurs qui ne pouvaient pas s'inscrire dans l'institution étaient de fait poussés aux marges du champ artistique, au point de tenter une existence autonome (à travers l'autoédition, comme le décrit Carole Hähnel-Mesnard ${ }^{14}$ ). C'est le cas de 1'auteur de théâtre Lothar Trolle $\left({ }^{*} 1944\right)$ que tu as fréquenté de près en tant que traducteur. Il a très tôt renoncé à une visibilité officielle pour s'engager dans les milieux parallèles, dans une forme de sous-culture.

13 Découvert par Brecht, Manfred Wekwerth (*1929) a dirigé le Berliner Ensemble de 1977 à 1991.

14 Carole HÄHNEL-MESNARD, La littérature autoéditée en RDA dans les années 1980 Un espace hétérotopique, Paris, L'Harmattan, 2007. 
MT : Oui, Lothar Trolle faisait aussi partie de la scène théâtrale berlinoise de la fin des années 1960, mais de manière dissimulée puisqu'il avait été embauché comme machiniste au Deutsches Theater. C'est comme ça qu'il avait pu voir les mises en scène importantes de Besson comme Edipe en 1967. À partir de 1969, il est devenu auteur de théâtre indépendant mais ses pièces n'étaient jouées nulle part. Pour subvenir à ses besoins, il s'occupait de la vente des produits du verger de sa mère. Il pouvait continuer à écrire pour lui, sans être admis à l'Union des écrivains de RDA. Il est devenu co-éditeur de la revue autoéditée Mikado dans les années 1980. Il n'était pas réellement dans une forme contre-culture car il n'avait pas de point d'accrochage avec le pouvoir. Il a plutôt mené une recherche formelle et poétique sur la langue. Lothar Trolle est pour moi une sorte de contrebandier des lois du marché et des frontières littéraires. Il ne s'inscrit pas vraiment dans un courant. D'une certaine façon, Trolle appartient à l'après-Müller, même s'il est son contemporain. Il refuse la linéarité dans l'écriture, avec ses textes-matériaux, il confronte le théâtre à la résistance du texte. En 1992, donc après la réunification, Frank Castorf a monté l'un de ses textes, Hermès dans la ville (Hermes in der Stadt), au Deutsches Theater. Trolle y fait dialoguer l'ancien et le nouveau, c'est une pièce où se télescopent les mythologies urbaines et antiques, les faits divers du quotidien et les épisodes bibliques, c'est une épopée sur la ville devenue banlieue. On ne sait pas vraiment si c'est du théâtre. C'est d'ailleurs un auteur qui est souvent considéré comme injouable. Ça ne l'empêche pas de poursuivre son chemin, de proposer des formes thêâtrales écrites dans et hors de l'institution. Il collabore encore aujourd'hui avec Castorf à la Volksbühne. Sans être dramaturge au sens fort du terme, il fournit des matériaux au metteur en scène pour confronter le texte de départ avec d'autres textes, il écrit en quelque sorte le «contre-texte », comme pour l'adaptation d'Alexandre Dumas Kean ou Désordre et Génie ${ }^{15}$.

Deux textes de Lothar Trolle ont été mis en espace lors du colloque de février 2012 à Toulouse, Après le déluge (Nach der Sintflut, 1978) et Le Dieu flâne (un des mouvements de Hermès dans la ville, 1991). Cette mise en espace a été possible grâce à la Compagnie La Vieille Dame et son metteur en scène Thomas Niklos.

Après le déluge est une clownerie qui donne à entendre et à voir que nous sommes dans la gadoue et que l'avenir tel qu'il se dessine est une traversée du désert. Quant au morceau Le Dieu flâne, il y apparaît que « le crime est toujours recommencé ». Le tout dans la joie et l'exubérance dada de la contre-culture. 


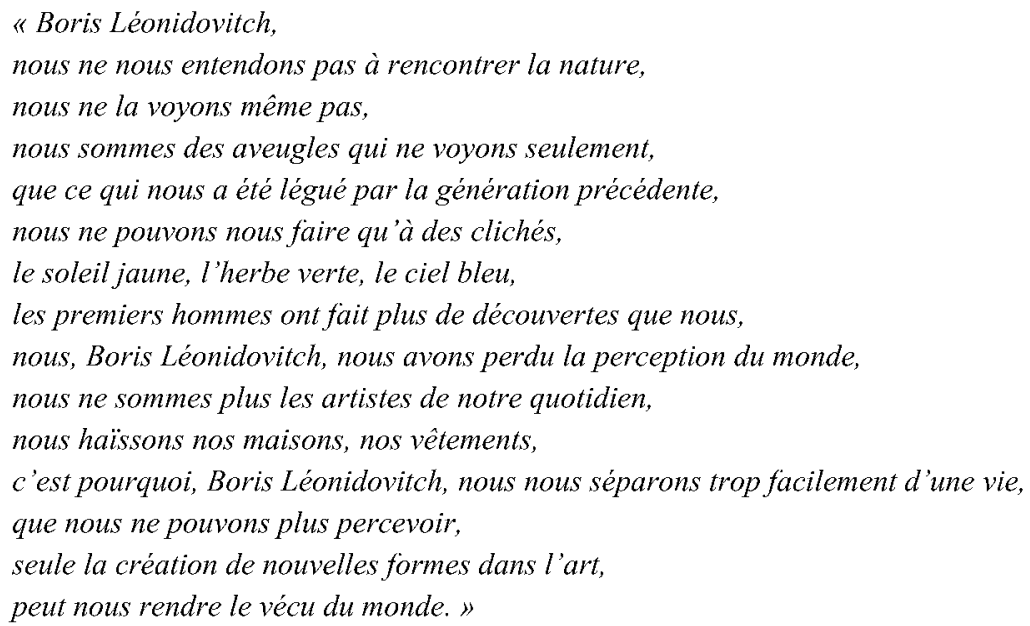

Extrait de Elles, à trois sous un pommier de Lothar Trolle, pièce traduite et mise en scène par Maurice Taszman en 2005. Il s'agit d'un discours prononcé par Viktor Schklovski lors des funérailles de Pasternak en 1914, réécrit par Lothar Trolle. 